\title{
Diagnóstico tardio de diabetes autoimune latente do adulto (LADA) em paciente idosa com episódios persistentes de hipoglicemias: relato de caso
}

\author{
Late diagnosis of latent adult autoimmune diabetes (LADA) in an elderly patient with persistent \\ episodes of hypoglycemia: case report \\ Diagnóstico tardío de diabetes autoinmune latente del adulto (LADA) en un paciente anciano con \\ episodios persistentes de hipoglucemia: reporte de caso
}

\author{
Bárbara Queiroz de Figueiredo \\ ORCID: https://orcid.org/0000-0003-1630-4597 \\ Centro Universitário de Patos de Minas, Brasil \\ E-mail: barbarafigueiredo@unipam.edu.br \\ Gabriel Augusto Batista Alves \\ ORCID: https://orcid.org/0000-0002-9915-1129 \\ Centro Universitário de Patos de Minas, Brasil \\ E-mail: gabrielaugusto84@ hotmail.com \\ Gardênia Silva Amorim \\ ORCID: https://orcid.org/0000-0001-7888-8562 \\ Centro Universitário de Atenas, Brasil \\ E-mail: gardênia.sa@hotmail.com \\ Larah Correia Borges \\ ORCID: https://orcid.org/0000-0001-5574-569X \\ Instituto Master de Ensino Presidente Antônio Carlos, Brasil \\ E-mail: 1cb_larah@hotmail.com \\ Luana Damaceno Miranda \\ ORCID: https://orcid.org/0000-0003-4013-2414 \\ Centro Universitário de Patos de Minas, Brasil \\ E-mail: luanad.m@hotmail.com \\ Vitória Figueiredo Garrido Cabanellas Nogueira \\ ORCID: https://orcid.org/0000-0003-4043-0534 \\ Universidade de Itaúna, Brasil \\ E-mail: vitoriafgcnogueira@gmail.com
}

\begin{abstract}
Resumo
Introdução: o conceito de diabetes autoimune latente do adulto, cuja sigla em inglês é LADA, de "latent autoimmune diabetes in adults" foi introduzido pela primeira vez em 1993, por Tuomi e Zimmet para definir pacientes diabéticos adultos que não requeriam insulina para controle da hiperglicemia nas fases iniciais da patologia, mas apresentavam autoanticorpos contra proteínas das células-beta e progressão mais rápida para necessidade de insulina. Objetivo: evidenciar um relato de caso de paciente idosa portadora de LADA que apresenta, frequentemente, episódios hipoglicêmicos. Metodologia: trata-se de um estudo de caso clínico com perspectiva qualitativa e descritiva, que consiste em uma pesquisa em que, em geral, ocorre com coleta direta de dados, cujo o pesquisador é o instrumento indispensável. Resultados e discussão: a hipoglicemia deflagra uma série de mecanismos contra-reguladores: suprime a secreção de insulina pelas células-beta, estimula a liberação de glucagon pelas células-alfa, a de adrenalina pela medula adrenal, além do cortisol e do hormônio de crescimento. Além disso, observam-se também a liberação de noradrenalina de neurônios simpáticos pós-ganglionares e acetilcolina dos pósganglionares simpáticos e parassimpáticos, além de outros neuropeptídeos Conclusão: os portadores de diabetes melito tipo 1 têm, com frequência, episódios de hipoglicemia durante a insulinoterapia, que, além do desconforto e de proporcionar situações constrangedoras no dia-a-dia, impedem a obtenção do controle glicêmico ideal. Mais ainda, hipoglicemias induzem deficiente mecanismo de contra-regulação em episódio posterior, com diminuição de liberação de adrenalina e dos sintomas de alarme, estabelecendo a síndrome de hipoglicemia associada à insuficiência autonômica.
\end{abstract}

Palavras-chave: LADA; Hipoglicemia; Diabetes mellitus tipo 1; Insulina; Glucagon.

\footnotetext{
Abstract

Introduction: The concept of latent autoimmune diabetes in adults, whose acronym in English is LADA, for "latent autoimmune diabetes in adults" was first introduced in 1993 by Tuomi and Zimmet to define adult diabetic patients
} 
who did not require insulin to control their hyperglycemia in the early stages of the pathology, but they presented autoantibodies against beta-cell proteins and faster progression to the need for insulin. Objective: to show a case report of an elderly patient with LADA who frequently presents hypoglycemic episodes. Methodology: this is a clinical case study with a qualitative and descriptive perspective, which consists of a research that, in general, takes place with direct data collection, in which the researcher is the indispensable instrument. Results and discussion: hypoglycemia triggers a series of counter-regulatory mechanisms: it suppresses insulin secretion by beta cells, stimulates glucagon release by alpha cells, adrenaline release by the adrenal medulla, in addition to cortisol and growth hormone. In addition, it is also observed the release of noradrenaline from postganglionic sympathetic neurons and acetylcholine from sympathetic and parasympathetic postganglionic neurons, in addition to other neuropeptides. Conclusion: patients with type 1 diabetes mellitus often have episodes of hypoglycemia during insulin therapy, which, in addition to discomfort and embarrassing situations in daily life, prevents the achievement of ideal glycemic control. Furthermore, hypoglycemia induces a deficient counter-regulation mechanism in a later episode, with decreased adrenaline release and alarm symptoms, establishing the hypoglycemia syndrome associated with autonomic failure.

Keywords: LADA; Hypoglycemia; Type 1 Diabetes mellitus; Insulin; Glucagon.

\begin{abstract}
Resumen
Introducción: el concepto de diabetes autoinmune latente en adultos, cuyo acrónimo en inglés es LADA, por "diabetes autoinmune latente en adultos" fue introducido por primera vez en 1993 por Tuomi y Zimmet para definir a los pacientes diabéticos adultos que no necesitaban insulina para controlar su hiperglucemia en las primeras etapas de la patología, pero presentaron autoanticuerpos contra las proteínas de las células beta y una progresión más rápida a la necesidad de insulina. Objetivo: presentar un caso clínico de un paciente anciano con LADA que presenta con frecuencia episodios de hipoglucemia. Metodología: se trata de un estudio de caso clínico con perspectiva cualitativa y descriptiva, que consiste en una investigación que, en general, se realiza con recolección directa de datos, en la que el investigador es el instrumento indispensable. Resultados y discusión: la hipoglucemia desencadena una serie de mecanismos contrarreguladores: suprime la secreción de insulina por las células beta, estimula la liberación de glucagón por las células alfa, la liberación de adrenalina por la médula suprarrenal, además de cortisol y hormona del crecimiento. Además, también se observa la liberación de noradrenalina de las neuronas simpáticas posganglionares y de acetilcolina de las neuronas posganglionares simpáticas y parasimpáticas, además de otros neuropéptidos. Conclusión: los pacientes con diabetes mellitus tipo 1 suelen presentar episodios de hipoglucemia durante la terapia con insulina, que en Además de las molestias y situaciones embarazosas de la vida diaria, impide la consecución de un control glucémico ideal. Además, la hipoglucemia induce un mecanismo de contrarregulación deficiente en un episodio posterior, con disminución de la liberación de adrenalina y síntomas de alarma, estableciendo el síndrome de hipoglucemia asociado a insuficiencia autonómica.
\end{abstract}

Palabras clave: LADA; Hipoglucemia; Diabetes mellitus tipo 1; Insulina; Glucagón.

\title{
1. Introdução
}

O diabetes mellitus (DM) é uma condição crônica de saúde muito prevalente em todos os países. A Organização Mundial de Saúde (OMS) estima que mais de 500 milhões de pessoas vivem, atualmente com DM em todo o mundo. A classificação atual do DM é baseada nos mecanismos fisiopatológicos que levam à hiperglicemia. No DM do tipo 1 (DM1) ocorre deficiência total ou quase total da produção de insulina pelas células beta das ilhotas pancreáticas enquanto no DM do tipo 2 (DM2) o que predomina é a resistência à ação da insulina nos tecidos associada a graus variáveis de deficiência da produção pancreática da mesma (Sociedade Brasileira de Diabetes, 2020).

O conceito de diabetes autoimune latente do adulto, cuja sigla em inglês é LADA, de latent autoimmune diabetes in adults foi introduzido pela primeira vez em 1993, por Tuomi e Zimmet para definir pacientes diabéticos adultos que não requeriam insulina para controle da hiperglicemia nas fases iniciais da patologia, mas apresentavam autoanticorpos contra proteínas das células-beta e progressão mais rápida para necessidade de insulina. Trata-se de uma forma de diabetes em que a autoimunidade contra as células beta pancreáticas se desenvolve de maneira mais arrastada e a obrigatoriedade do uso da insulina pode se dar após vários de diagnóstico. No LADA, a disfunção da célula $\beta$ tem sido reportada como intermediária entre os dois principais tipos de diabetes (DM1 e DM2) (Calsolari et al. 2007).

O LADA, por definição, acomete pessoas de 25 anos ou mais, mas pode incidir em qualquer idade fazendo com que, muitas vezes, o paciente seja diagnosticado como tendo DM2. Do ponto de vista fisiopatológico, o DM1 resulta da falta 
absoluta ou quase absoluta de insulina enquanto o DM2 ocorre por resistência à ação da insulina nos tecidos e graus variáveis de redução na produção do hormônio. Fenômenos genéticos, imunológicos e metabólicos parecem convergir com outros processos de doença condicionando a apoptose da célula $\beta$ e contribuindo para o aparecimento da LADA. De acordo com Jones et al. (2021), sendo ela identificada com um grupo de características clínicas e genéticas intermediárias entre os dois tipos de diabetes, nota-se que esse fenômeno ainda não está claro, sendo propostas, pelo autor, três teorias: como um fenótipo mais brando que inicia-se em idades mais tardias e que são semelhantes ao DM2, mas que requer insulina; como uma síndrome em que as fisiopatologias do DM1 e DM2 estão presentes em cada paciente; como um grupo heterogêneo decorrente de dificuldades de classificação.

O diagnóstico de LADA é tipicamente baseado no achado de hiperglicemia, associado à constatação de que a causa seja devida à uma falha das ilhotas de Langherans em produzir insulina, presença de sintomas agudos, índice de massa corporal $<25 \mathrm{~kg} / \mathrm{m} 2$, história pessoal ou familiar de outras doenças autoimunes. A presença de, pelo menos, duas destas características clínicas justifica o pedido de anticorpos contra as células beta pancreáticas. Tem-se, atualmente, quatro tipos de anticorpos: anti-glutamato descarboxilase (GADA), anti-insulina (IAA), anticélulas beta (ICA) e antitransportador de zinco. No LADA, os anticorpos anti-insulina (IAA) são raros, enquanto os GADA são característicos, mas estes podem se tornar negativos ao longo do tempo. Indivíduos com título elevado de GADA ou com positividade para maior número de anticorpos, geralmente apresentam um índice de massa corporal (IMC) inferior, menor secreção endógena de insulina e progressão mais rápida para insulinoterapia. Assim, o título de GADA pode permitir identificar indivíduos que respondem pior à terapêutica com antidiabéticos orais e que apresentam maior risco de cetoacidose (Silva, 2007).

A detecção de um nível baixo de peptídeo-C e anticorpos contra as ilhotas de Langerhans reforçam e sustentam este diagnóstico. Uma diferenciação com o diabetes tipo 2 pode ser feita porque as pessoas com LADA tipicamente têm níveis baixos, embora às vezes moderados, de peptídeo $\mathrm{C}$ à medida que a doença progride, e aqueles com resistência à insulina ou diabetes tipo 2 são mais propensos a ter níveis elevados de peptídeo $\mathrm{C}$ devido a uma produção excessiva de insulina. Clinicamente, os indivíduos com LADA representam um grupo heterogêneo de doentes com títulos variáveis de anticorpos, índice de massa corporal (IMC) e progressão para insulinoterapia (Couri et al. 2009).

A hipoglicemia é definida pelo desenvolvimento de sintomas autonômicos ou neuroglicopênicos (Tabela 1), um baixo nível de glicose plasmática ( $<4,0 \mathrm{mmol} / \mathrm{L}$ para pessoas com diabetes tratadas com insulina ou secretagogo de insulina); e sintomas que respondem à administração de carboidratos (Guisaola et al. 2010). A gravidade da hipoglicemia é definida pelas manifestações clínicas (Tabela 2). A hipoglicemia é mais comum em pessoas com diabetes mellitus de tipo 1, seguida por pessoas com diabetes mellitus tipo 2 tratada com insulina e pessoas com Diabetes mellitus tipo 2 controlada por antidiabéticos.

Tabela 1: Sintomas de hipoglicemia.

\begin{tabular}{|l|l|}
\hline \multicolumn{1}{|c|}{ NEUROGÊNICO (AUTÔNOMO) } & \multicolumn{1}{c|}{ NEUROGLICOPÊENCO } \\
\hline Palpitações trêmulas & Dificuldade de concentração \\
\hline Sudorese & Confusão mental e dor de cabeça \\
\hline Ansiedade & Fraqueza \\
\hline Fome & Sonolência e tontura \\
\hline Náusea & Alterações visuais \\
\hline Formigamento & Dificuldade para falar \\
\hline
\end{tabular}

Fonte: Yale et al (2018). 
Tabela 2: Gravidade da hipoglicemia.

\begin{tabular}{|l|l|l|}
\hline \multicolumn{1}{|c|}{ LEVE } & \multicolumn{1}{|c|}{ MODERADA } & \multicolumn{1}{c|}{ GRAVE } \\
\hline Sintomas autonômicos presentes & $\begin{array}{l}\text { Sintomas autonômicos } \\
\text { neuroglicopênicos presentes }\end{array}$ & Pode ocorrer inconsciência \\
\hline O indivíduo é capaz de tratar-se & O indivíduo é capaz de tratar-se & $\begin{array}{l}\text { O indivíduo requer assistência de } \\
\text { terceiro }\end{array}$ \\
\hline
\end{tabular}

Fonte: Yale et al (2018).

Os principais fatores de risco para hipoglicemia grave em pessoas com diabetes tipo 1 incluem um episódio anterior de hipoglicemia grave, hemoglobina de baixo glicose atual (A1C) $(<6,0 \%)$, desconhecimento da hipoglicemia, diabetes de longa duração, neuropatia autonômica, adolescência e crianças em idade pré-escolar incapazes de detectar e / ou tratar a hipoglicemia leve por conta própria. Os fatores de risco para hipoglicemia em pessoas com diabetes tipo 2 incluem o avanço da idade, comprometimento cognitivo grave, baixa alfabetização em saúde, insegurança alimentar, duração da terapia com insulina, insuficiência renal e neuropatia (Yale et al. 2018).

Indivíduos com alto risco de hipoglicemia grave, devem ser informados de seus riscos e aconselhados, juntamente com seus entes, sobre como prevenir e tratar a hipoglicemia (incluindo o uso de glucagon), prevenir acidentes de trânsito e industriais por meio do automonitoramento da glicose no sangue e tomar as precauções adequadas antes da atividade e documentar as leituras de glicose no sangue feitas durante as horas de sono. Os indivíduos podem precisar ter seu regime de insulina ajustado adequadamente para reduzir o risco (Berlin et al. 2005).

A hipoglicemia frequente pode diminuir as respostas normais à hipoglicemia e levar a uma contra-regulação da glicose defeituosa e desconhecimento da hipoglicemia. O desconhecimento da hipoglicemia ocorre quando o limiar para o desenvolvimento de sintomas de alerta autonômicos é próximo ou inferior ao limiar para os sintomas neuroglicopênicos, de modo que o primeiro sinal de hipoglicemia é confusão ou perda de consciência. A hipoglicemia grave é muitas vezes a barreira primária para atingir as metas glicêmicas em pessoas com diabetes tipo 1 e ocorre frequentemente durante o sono ou na presença de desconhecimento de hipoglicemia. A resposta simpatoadrenal à hipoglicemia é reduzida durante o sono e após exercícios ou consumo de álcool (Schultes et al. 2007).

A hipoglicemia noturna assintomática é comum e geralmente dura mais de 4 horas. A hipoglicemia grave, resultando em convulsões, é mais provável de ocorrer à noite do que durante o dia. Tanto o desconhecimento da hipoglicemia, quanto a contra-regulação da glicose em níveis errôneos são potencialmente reversíveis. Evitar estritamente a hipoglicemia por um período de 2 dias a 3 meses foi associado à melhora no reconhecimento de hipoglicemia grave, as respostas hormonais contrarregulatórias ou ambos (Yeoh et al. 2015). Sob essa perspectiva, o objetivo desta pesquisa é evidenciar um relato de caso de paciente idosa portadora de LADA que apresenta, frequentemente, episódios hipoglicêmicos.

\section{Metodologia}

Este artigo trata-se de um estudo de caso clínico com perspectiva qualitativa e descritiva, que consiste em uma pesquisa em que, em geral, ocorre com coleta direta de dados, cujo o pesquisador é o instrumento indispensável. O presente estudo contou com o aporte de trabalhos acadêmicos entre os anos de 2001 e 2020. Por meio do acesso às principais bases de dados, a saber: dados National Library of Medicine (PubMed MEDLINE), Scientific Electronic Library Online (Scielo), Cochrane Database of Systematic Reviews (CDSR), Google Scholar, Biblioteca Virtual em Saúde (BVS) e EBSCO Information Services, no período compreendido entre março e julho de 2021. O Termo de Consentimento Livre e Assistido (TCLE) foi assinado pelo paciente para coleta de dados neste estudo. 


\section{Relato de Caso}

Trata-se de paciente do sexo feminino, 65 anos de idade e diagnóstico de diabetes autoimune latente (LADA) em abril de 2019, que se justificou por episódios de hiperglicemias e correlação empírica com doença autoimune já existente na família, posteriormente à dosagem de anticorpos GADA contra as células beta pancreáticas, com marcação de 2000,0 UI/mL (VR: inferior a 10,0 UI/mL), bem como dosagem de hemoglobina glicada, que constou 9,3\%. Dada a identificação, a patologia seguiu-se com controle por meio de modificações dietéticas, administração insulínica de ação intermediária e lenta, a Lantus, e de ação rápida, a Asparte Fiasp, cuja dosagens variam de acordo com refeições, bem como uso diário de empagliflozina/linagliptina, ou seja, o medicamento Glyxambi, a fim de melhor controle glicêmico e melhora da qualidade de vida.

A paciente relata episódios frequentes de hipoglicemia durante a insulinoterapia, principalmente durante a noite, com sintomas neuroglicopênicos típicos como tontura, fraqueza e alterações visuais, aproximadamente uma vez a quatro dias. Desse modo, foi avaliada para adequação da medicação e orientações para maior adesão ao controle da glicemia por meio da frequente medição, além de maior atenção à medicação, que também deve ser fracionada.

\section{Discussão}

A hipoglicemia deflagra uma série de mecanismos contra-reguladores: suprime a secreção de insulina pelas célulasbeta, estimula a liberação de glucagon pelas células-alfa, a de adrenalina pela medula adrenal, além do cortisol e do hormônio de crescimento. Além disso, observam-se também a liberação de noradrenalina de neurônios simpáticos pós-ganglionares e acetilcolina dos pós-ganglionares simpáticos e parassimpáticos, além de outros neuropeptídeos (Ahrén et al. 2000).

A queda da secreção de insulina possibilita aumentar a produção hepática e renal de glicose, além de diminuir sua captação nos tecidos periféricos, especialmente muscular. O glucagon tem papel fundamental nesse mecanismo, aumentando a glicogenólise hepática e favorecendo a gliconeogênese. A liberação de adrenalina resulta maior produção hepática de glicose e diminuição de captação nos tecidos insulino-sensíveis, além de ajudar na percepção dos sintomas e contribuir para diminuição de secreção de insulina por mecanismo a-adrenérgico. Seu papel torna-se crítico quando a secreção de glucagon é insuficiente (Taborsky et al. 2002).

Em portadores de DM1 não ocorre diminuição da secreção de insulina em resposta à hipoglicemia, uma vez que sua concentração circulante, causadora de hipoglicemia, depende da absorção da insulina administrada. A resposta esperada, de aumento na secreção de glucagon na vigência de hipoglicemia, deixa de existir precocemente no curso da doença em portadores de DM1 (Gosmanov et al. 2005). Várias hipóteses tentam explicar esse evento: alteração na arquitetura da ilhota, neuropatia autonômica, alteração no receptor de glicose na célula-alfa (Kishore et al. 2006). No entanto, embora esses fatores possam contribuir, certamente não pode haver um fator único: assim, sabe-se que há liberação de glucagon a outros estímulos em portadores de DM1, o que sugere um problema com a sinalização e não da estrutura da célula-alfa (Woerle et al. 2003).

A adrenalina e o glucagon estimulam a glicogenólise e a gliconeogênese, e a interação entre a metabolização do glicogênio e a gliconeogênese, em harmonia, possibilita rápida recuperação de hipoglicemia. Estudo recente, analisando a produção hepática de glicose e medida de glicogênio hepático por espectroscopia, verificou que, com efeito, em portadores de DM1, tratados intensivamente, a produção endógena de glicose diminui principalmente por glicogenólise deficiente (Bingham et al. 2005).

\section{Conclusão}

Os portadores de Diabetes mellitus tipo 1 têm, com frequência, episódios de hipoglicemia durante a insulinoterapia, que, além do desconforto e de proporcionar situações constrangedoras no dia-a-dia, impedem a obtenção do controle glicêmico 
ideal. Mais ainda, hipoglicemias induzem deficiente mecanismo de contra-regulação em episódio posterior, com diminuição de liberação de adrenalina e dos sintomas de alarme, estabelecendo a síndrome de hipoglicemia associada à insuficiência autonômica. A ocorrência de hipoglicemias durante algumas atividades de risco, em especial a direção veicular, pode resultar acidentes com o paciente e terceiros, além de lesão de propriedade, motivo pelo qual pessoas com diabetes devem ser orientadas quanto aos cuidados na direção de veículos. Em geral, a recuperação neurológica é total após a correção de coma hipoglicêmico. No entanto, quando esses episódios são repetitivos, especialmente em crianças, podem ter como consequência distúrbios cognitivos definitivos. A reversão de quadros de hipoglicemia sem sinal de alerta é difícil, devendo-se evitar meticulosamente sua ocorrência, adequando o tratamento, os alvos glicêmicos, utilizando a monitoração domiciliar e fazendo treinamento para o reconhecimento precoce de hipoglicemias.

\section{Referências}

Ahrén, B. (2000). Autonomic regulation of islet hormone secretion-implications for health and disease. Diabetologia, 43(4), 393-410.

Berlin, I., Sachon, C. I., \& Grimaldi, A. (2005). Identification of factors associated with impaired hypoglycaemia awareness in patients with type 1 and type 2 diabetes mellitus. Diabetes Metab., 31 (3), 246-251

Bingham, E. M., Dunn, J. T., Smith, D., Sutcliffe-Goulden, J., Reed, L. J. \& Marsden P. K. (2005). Differential changes in brain glucose metabolism during hypoglycaemia accompany loss of hypoglycaemia awareness in men with type 1 diabetes mellitus. Diabetologia, 48 (10), $2080-2089$.

Calsolari, M. R., Rosário, P. W. S., Reis, J. S., Silva, S. C. \& Purisch, S. (2008). Diabetes autoimune latente do adulto ou diabetes melito tipo 2 magro? Arq. Bras. Encodrinol. Metab., 52 (2), 1-6.

Couri, C. E., Oliveira, M. C. B., Stracieri, A. B. P. L., Moraes, D. A. \& Voltarelli, J. C. (2009). C-peptide levels and insulin independence following autologous nonmyeloablative hematopoietic stem cell transplantation in newly diagnosed type 1 diabetes mellitus. JAMA, 301 (15), $1573-1579$.

Gosmanov, N. R., Szoke, E., Israelian, Z., Smith, T., Cryer, P. E. \& Gerich, J. (2005). Role of the decrement in intraislet insulin for the glucagon response to hypoglycemia in humans. Diabetes Care. 28 (3), 124-131.

Guisasola, F. A., Yin, D. D. \& Nocea, G. (2010). Associação de sintomas hipoglicêmicos com a avaliação dos pacientes sobre seu estado de qualidade de vida relacionada à saúde: um estudo transversal. Resultados de Saúde Qual Life, 8 (86).

Jones, A. G., McDonald, T. J., Shields, B. M., Hagopian, W. \& Hattersley, A. T. (2021). Latent Autoimmune Diabetes of Adults (LADA) is likely to representant a mixed population of autoimmune (type 1) and nonautoimmune (type 2) diabetes. Diabetes Care, 44 (1), 1-9.

Kishore, P., Gabriely, I., Cui, M. H., Di Vito, J., Gajavelli, S. \& Hwang, J. H. (2006). Role of hepatic glycogen breakdown in defective counterregulation of hypoglycemia in intensively treated type 1 diabetes. Diabetes, 55(3), 659-666.

Schultes, B., Jauch-Chara, K. \& Gais, S. (2007). Defective awakening response to nocturnal hypoglycemia in patients with type 1 diabetes mellitus. PLoS Med., 4 (69), 1-5.

Silva, M. E. R. (2007). Precisamos diagnosticar o diabetes Latente Autoimune do Adulto (LADA)? Arq. Bras. Encodrinol. Metab., 51 (1).

Sociedade Brasileira de Diabetes (SBD) (2020). Diretrizes da Sociedade Brasileira de Diabetes: 2020-2021. Clannad.

Taborsky, G. J., Ahren, B., Mundinger, T. O., Mei, Q. \& Havel, P. J. (2002). Autonomic mechanism and defects in the glucagon response to insulin-induced hypoglycaemia. Diabetes Nutr Metab., 15 (3), 318-322

Woerle, H. J., Meyer, C., Popa, E. M., Cryer, P. E. \& Gerich, J. E. (2003). Renal compensation for impaired hepatic glucose release during hypoglycemia in type 2 diabetes: further evidence for hepatorenal reciprocity. Diabetes, 52(6):1386-1392.

Yale, J. F., Paty, B. \& Senior, P. A. (2018). Hypoglycemia. Canadian Journal of Diabetes, 42 (1), 104-108.

Yeoh, E., Choudhary, P. \& Nwokolo, M. (2015). Interventions that restore awareness of hypoglycemia in adults with type 1 diabetes: A systematic review and meta-analysis. Diabetes Care, 38 (8), 1592-1609 